ECOLOGY AND SOCIETY

Home | Archives | About | Login | Submissions | Notify | Contact | Search

ES HOME > VOL. 5, NO. 1 > ART. 6

Copyright (c) 2001 by The Resilience Alliance

The following is the established format for referencing this article:

Thomson, J. D. 2001. Using pollination deficits to infer pollinator declines: Can theory guide us? Conservation

Ecology 5(1): 6. [online] URL: http://www.consecol.org/vol5/iss1/art6/

A version of this article in which text, figures, tables, and appendices are separate files may be found by following this link.

Synthesis, part of Special Feature on Pollinator Decline

\title{
Using Pollination Deficits to Infer Pollinator Declines: Can Theory Guide Us?
}

\author{
James D. Thomson
}

\section{University of Toronto}

- Abstract

- Dedication

- Introduction

- Issues Related to Pollination Deficits

O How are deficits measured?

Empirical evidence for deficits

Theoretical aspects of deficits

Problems in assessing pollination deficits

Nonlinear effects of pollinator visitation rate

O Putting pollination deficits into the context of plant life histories

Does life-history evolution set up patterns that serve as signatures of pollinator decline?

- Elements of a Thorough Study of Pollination Deficits

- Suggestions for Future Research

- Responses to this Article

- Acknowledgments

- Literature Cited

\section{ABSTRACT}

Authors examining pollinator declines frequently discuss pollination deficits, either as contemporary evidence that declines have occurred or as a possible negative consequence of future declines. Because pollination deficits can be measured in short-term studies, it would be useful if such studies could somehow replace painstaking documentation of insect population trends. I examine the legitimacy of this type of substitution with reference to evolutionary theory and natural plant populations. Operationally, pollination deficits are detected through pollen supplementation experiments. Although simple, these experiments are subject to subtleties of interpretation because of biases and nonlinear responses, which I discuss. Although it has been found that, in $62 \%$ of the natural populations studied, fruit or seed sets are at least sometimes limited by insufficient pollen, other research suggests that intact natural systems ought to arrive at an evolutionary equilibrium in which reproduction is limited equally by pollination and by maternal resources. Therefore, chronic severe pollination deficits may indicate that the pollinator service of a plant population has declined from some higher level in the past. However, there is no evidence of widespread declines, and, because of stochastic factors in nature, occasional shortfalls of pollination should be expected even at equilibrium. Although the effects of pollination deficits on plant population dynamics have been little studied, moderate declines in seed production may have relatively little effect on population growth rates because resources not expended on fruits and seeds may be reallocated to vegetative persistence or spread. It is therefore premature to conclude that pollinator declines are having strong effects on natural plant populations, but 
this mostly reflects a lack of data and is no cause for complacency. Theory must be supplemented by case studies; I give one example and recommendations.

KEY WORDS: Bateman's principle, Primula sieboldii, reproductive cost, fruit and seed set limits, pollen presentation theory, pollen removal and deposition, pollination deficit, pollinator decline, pollinators, resource allocation, resource limitation, supplemental pollination, supplemental pollination, evolutionary equilibrium.

Published: April 5, 2001

\section{DEDICATION}

Dedicated to F. James Rohlf on the occasion of his 65th birthday.

\section{INTRODUCTION}

Claims of widespread declines in populations of animal pollinators have recently captured public and scientific attention. In most of what has been written on "pollinator declines," decreases in the size of animal populations have received the most coverage in theoretical or conceptual discussions. However, the size of an animal population is difficult to measure, and this is especially true of insects, which are the most important pollinators in most habitats. Therefore, when the discussion turns to hard evidence, the emphasis often shifts to "pollination deficits" suffered by plants (Kearns et al. 1998). Indeed, shortages of pollinators and shortfalls of seed or fruit production are really two facets of the same problem. Long ago, Rachel Carson pointed out that silent springs and fruitless falls go hand in hand (Buchmann and Nabhan 1996: 23). Furthermore, the loss of plant fecundity figures prominently in arguments about why the public should be concerned about pollinator declines (Buchmann and Nabhan 1996).

Other articles in this collection discuss direct evidence for declines in the population sizes, densities, or ranges of pollinating animals. Here, I consider whether studies of pollination deficits themselves can be used as surrogates for studies of pollinator declines. I shall briefly consider whether there is possible evidence of general shortages of animal pollinators, but my main aim is to clarify some of the logical and logistical issues that arise when considering pollination deficits as evidence of pollinator declines. To avoid repeating the phrase "seed set or fruit set," I will use the term "fecundity" to cover both modes of female reproductive success. I discuss one case study that does a particularly good job of tying fecundity shortfalls to pollination, and I derive some recommendations for further studies.

\section{ISSUES RELATED TO POLLINATION DEFICITS}

\section{How are deficits measured?}

Two procedures are commonly used to infer deficits. Evolutionary ecologists typically conduct "supplemental pollinations" in which a set of randomly chosen control plants is exposed to natural levels of pollination, while a matched set of supplemented plants receives hand pollination in addition to whatever unaided pollination it receives naturally. In contrast, agricultural experiments are more likely to involve supplementing the number of pollinators, for example, by bringing hives of bees to a test field, and comparing the fecundity of the supplemented field to that of a control field. Although in this essay I will not dwell on the latter approach, the contrast between these two methods of inferring deficits is relevant to the question of how to define the concept of a "deficit." Obviously, this term implies a shortfall in fecundity relative to some benchmark that in turn represents full or complete pollination. In practice, these benchmarks are determined through particular experimental or observational procedures; for this reason, the definition of a deficit must be an operational one.

One way in which these procedures differ is that supplemental pollinations are frequently done with pure outcross pollen, whereas real pollinators almost always deliver a mixture of self, geitonogamous, and outcross pollen. Therefore, for many-flowered plants that benefit from outcrossing, the level of fecundity achieved by hand pollination may represent an ideal condition that is unobtainable by any real animal visitor. As a benchmark for comparison, it represents, at least in principle, a "perfect" pollination. Although pollinator manipulations are therefore somewhat more "realistic" in that they represent a state that could actually be achieved in the real world, they can seldom be done at enough replicate sites to provide statistical power. Sampling multiple plants or flowers within sites typically provides only pseudoreplication. There are a number of subtle points involved in interpreting these experiments, which I will consider after looking at three surveys on the extent of pollinator limitation.

\section{Empirical evidence for deficits}


Burd's (1994) review of the literature on hand-pollination experiments identified usable studies of 258 wild plant species from 77 families; he deliberately excluded cultivated plants. Although results varied depending on the response variable measured, Burd found that pollination limitation was frequent. Of the 258 species studied, $62 \%$ showed significant limitation of some sort. For species in which the number of seeds per plant was recorded, seven of eight species $(87.5 \%)$ were limited. For cases in which the number of seeds per fruit was recorded, only $28 \%$ of 86 plants showed limitation.

Mayfield (1998) extended Burd's approach to include hand-pollination experiments on cultivated crops. As mentioned above, hand-pollination experiments are rare on such plants, so she found only 12 papers on 16 cultivars of 11 crop species. However, these studies reported similar frequencies of pollination limitation (59\% compared to Burd's 62\%). Mayfield concluded that, although the evidence is sparse, both cultivated and wild plants frequently labor under pollination deficits. For cultivated species, this conclusion is reinforced by McGregor's (1976) compilation, which gives crop-specific recommendations for increasing pollination for the great majority of species examined.

On the other hand, V. J. Tepedino and associates (Tepedino et al. 1999; V. J. Tepedino, unpublished data) have been carrying out supplemental pollinations of many entomophilous native plant species that are conservation targets in the western United States. With a present sample size of more than 30 species, they have found pollinator limitation to be uncommon (10-15\% of cases), even though these are plants that typically have small or fragmented populations and might therefore be at high risk for pollination deficits ( $\mathrm{V}$. J. Tepedino, personal communication). Although, as explained below, there are a number of caveats regarding the interpretation of supplementation experiments, there seems to be a difference between Burd's literature review and Tepedino's systematic studies. One possibility is that, despite the rarity of Tepedino's study plants, the region in which he works may retain a healthier pollinating fauna than many of the heterogeneous sites in which the studies in Burd's review were carried out. The only firm conclusion is that some wild and cultivated plant populations do show indications of pollination deficits, and it would appear that these deficits are not especially rare.

\section{Theoretical aspects of deficits}

If we consider Burd's broad review to represent the state of the world in general, can we say that the high frequency of pollination deficits might signal a decline in pollinator populations? One line of reasoning would agree: Willson (1979) invoked Bateman's principle, which posits that the sex that invests more in the production of offspring becomes a "resource" for which members of the less parental sex compete. On the basis of this principle, Willson argued that one would expect a plant's female function (seed production) to be limited by resources, not by pollination, whereas male function (pollen dissemination) ought to be limited by pollination. Many authors further developed this theme (e.g., Charnov 1982, Bell 1985), leading to the consensus that many floral characters are best interpreted as adaptations for male reproductive success. If Bateman's principle really is an accurate description of plant reproduction, then pollination ought to have little effect on female fecundity in an equilibrial "state of nature." In that case, widespread pollination deficits, such as those recorded by Burd, might indeed indicate that something had gone out of balance.

However, the application of Bateman's principle to plants has been criticized as simplistic. Wilson et al. (1994) argue in favor of an alternative suggestion by Haig and Westoby (1988) that natural selection should, in fact, lead populations toward a balance point between chronic resource limitation and chronic pollination limitation. This balance is achieved through the allocation of resources to different functions. If plants are chronically underpollinated, then selection will favor variants that allocate less to pistils and more to pollinator attractants or rewards. If pollination is seldom limiting, variants that reallocate resources from attractants to pistils will be favored. In this view, most plant species would show pollination deficits, but only in some sites and some seasons, as pollinator populations fluctuated stochastically around long-term averages. If Haig and Westoby's view of plant reproduction is correct, pollination deficits on the scale revealed by Burd's review would turn up frequently in the natural course of events; they would not provide any basis for inferring that pollinators have declined in general.

On the other hand, if Haig and Westoby's principle applies, we would not expect any particular plant population to be chronically pollinator-limited, season after season. Finding such a plant population would, in fact, suggest that the plant's current pollination regime had deteriorated from a former long-term average that had molded the plant's current allocational balance over recent evolutionary time. Therefore, this principle does suggest to us a way of inferring pollinator declines from pollination deficits, although the argument is based on a very simple notion of optimal allocation, and does not immediately lead to quantitative rules for decision making. For example, if a study population is significantly pollination limited in eight of 10 seasons, how confident should we be that pollinators have declined from some higher level in the past? In a plant population with a seed bank, a tendency to overproduce flowers in most years might possibly be an adaptive tactic designed to take full advantage of the years when pollinator numbers are particularly high (I. Parker, personal communication). Critical thought and modeling are needed to develop and evaluate the practical applicability of the Haig-Westoby notion.

\section{Problems in assessing pollination deficits}


I referred above to the application of pure outcross pollen as an "unrealistic" reference point for assessing pollination deficits. From the standpoint of applying the Haig and Westoby (1998) argument, a better reference point would be the best pollination service that could be obtained from natural animal pollination, but it is not clear that any particular regime of hand pollination is able to mimic the "best natural" level. I wish to point out four other problems associated with the conduct and interpretation of pollination deficit assessments.

First, as noted by Wilson et al. (1994), researchers tend to carry out such experiments at their primary study sites, and those sites tend to be selected for study because they contain relatively large, healthy populations of the plant of interest. This introduces a bias. Even if the selected sites show no indications of pollinator limitation, the same is not necessarily true of smaller, peripheral populations.

Second, the notion of "no pollen limitation" is actually a dubious proposition. Like everything else in our variable world, stigma loads of pollen will vary, possibly according to some statistical distribution. Even when the mean stigma load is plentiful enough to fertilize all ovules, some flowers will by chance receive smaller loads that may fail to set all possible seeds. By this argument, we expect that there will always be some reduction of fecundity due to insufficient pollination, i.e., there is always some pollen deficit. The question really becomes one of statistical power. How small a pollination deficit can be detected with a given experimental design?

Table 1. Power tests (via simulation) for pollen supplementation experiments. Tabulated values are the numbers of nonsignificant $(P>0.05) t$ tests out of 100 iterations for various sample sizes at various levels of actual pollination limitation.

\begin{tabular}{|c|c|c|c|}
\hline $\begin{array}{c}\text { Fertilized } \\
\text { ovules } \\
\text { (\%) }\end{array}$ & \multicolumn{3}{|c|}{ Sample size } \\
\cline { 3 - 4 } & 20 & 40 & 80 \\
\hline 95.1 & 93 & 66 & 5 \\
\hline 92.1 & 81 & 23 & 0 \\
\hline 90.2 & 75 & 13 & 0 \\
\hline 85.0 & 47 & 1 & 0 \\
\hline
\end{tabular}

Table 1 represents a power analysis, via simulation, for a simple case of normally distributed stigma loads and a sigmoidal relationship between stigma load and number of ovules fertilized. Moderate pollination deficits of up to $15 \%$ will usually not be detected with sample sizes of 20 individuals, and even 40 are insufficient for small deficits. Whether small deficits are important to a particular plant population is a question that requires demographic analysis. Whether they are important to evaluating departures from a Haig-Westoby equilibrium is a question that requires more theory.

Third, Young and Young (1992) reviewed evidence that undermines an implicit assumption of pollen supplementation experiments, i.e., that more pollen is always better. Out of 99 cases, they found that hand pollination significantly increased fecundity over "natural" pollination in 42 cases, had no effect in 40 cases, and significantly decreased fecundity in 17 cases. The last category of results is puzzling, and raises doubts about the whole enterprise of hand pollinating. Young and Young suggest several possible reasons for these unexpected results. Too much pollen may actually be bad because of effects such as pollen tube crowding. Alternatively, hand pollination may introduce artifacts, such as mechanical damage to stigmas, pollination with unviable grains, etc. If artifacts are to blame, findings of "no pollination deficits" may be misleading; although more pollination could potentially benefit the plants, poor pollination technique might negate the potential advantage. If plants really do best at an optimal, intermediate intensity of pollination, several kinds of misinterpretation are possible. To eliminate these, pollination experiments would have to be redesigned to explore the effects of various pollination levels, as suggested by Young and Young (1992).

Fourth, there are many minor but important factors to consider in pollinator supplementation experiments. For practical reasons, they almost always add more individuals from a single pollinator species to a pre-existing system, rather than replacing one type of visitor with another. The implicit assumptions here are that (1) adding more pollinators leads to increased pollen transfer, and (2) increased pollen transfer is beneficial for plant fecundity. As seen above, Young and Young's (1992) compilation casts doubt on the second of these assumptions, and the first is also questionable. Because different pollinators interact with one another through their individual contributions 
toward the depletion of a common pool of pollen grains, pollinators are not simply additive in their effects on pollen delivery. For a complete understanding of how plant reproductive success varies with changes in pollinator populations, it is necessary to consider these nonlinearities.

\section{Nonlinear effects of pollinator visitation rate}

As the number of pollinator visits received by a plant increases from zero, the reproductive success of the plant is expected to rise initially but then to level off or possibly decline (Young 1988, Young and Young 1992). We tend to think that saturation occurs because all of the ovules have been fertilized, which is the classic case where resource limitation takes over from pollination as the determinant of female fecundity. However, other scenarios are possible. Because some pollinators, such as female bees, are avid collectors of pollen for feeding their broods, it is possible that pollen supplies become depleted before ovule fertilization is complete. Such effects depend on the balance between a pollinator's removal of pollen from anthers and its subsequent deposition of some of those grains on stigmas (Thomson and Thomson 1992).

Bees commonly remove $70-80 \%$ of the pollen that is presented in a flower, even when they are foraging for nectar without any special behaviors directed at pollen collection; probably less than $1 \%$ of the removed grains get deposited on stigmas (Harder and Thomson 1989). At such high removal rates, a flower's pollen can be exhausted after only a few visits. Therefore, its reproductive success may not continue to increase with increased visitation, but instead may saturate quickly because of pollen depletion. Furthermore, by depleting the available pollen, one visitor's activities reduce the ability of later visitors to contribute to the plant's reproductive success. The most interesting example of this is "conditional parasitism." In this case, a visitor species (A) that removes much pollen but deposits little of it can be a beneficial mutualist to a plant when it is the only visitor available. If, however, a second species of visitor (B) is also available that delivers a higher fraction of what it removes, then $A$ may be transformed from a mutualist when alone to a parasite when in the company of $B$, because $A$ removes grains from the system that would otherwise be delivered to stigmas by B. Different species of bees are, in fact, known to differ in their removal and deposition rates on the same plants (Wilson and Thomson 1991, Goodell and Thomson 1997; N. Williams and J. D. Thomson, unpublished manuscript).

Admittedly, interactions between pollinators through depletion of pollen are far more likely to affect male reproductive success than female fecundity. Even with low deposition rates and much pollen wastage, if pollinators are visiting frequently enough to deplete pollen supplies, stigma loads may still be adequate for high fruit and seed set. However, when flowers have many ovules, pollinators with very low deposition rates, e.g., pollen-collecting honey bees on Impatiens capensis (Wilson and Thomson 1991), may exhaust a flower's pollen supply without depositing enough pollen to fertilize all of its ovules. Further quantitative studies are needed in this area.

From the standpoint of interpreting pollinator supplementation experiments, pollen depletion models suggest two provocative possibilities. First, adding more individuals of an A-type (high-removal, low-deposition) pollinator will not necessarily increase fecundity, but adding more individuals of a B-type pollinator might. Second (and counterintuitively), removing A-type pollinators from a multipollinator system might increase pollen receipt and fecundity (Thomson and Thomson 1992). Indeed, good pollinators cannot achieve their highest potential service to plants if inferior pollinators are taking pollen out of circulation. These results suggest caution in implementing "common sense" management policies for improving pollination. Increasing the numbers of a pollinator could actually be detrimental unless it is the right pollinator. Ironically, certain types of specialized pollinators, such as oligolectic solitary bees, may be so good at collecting pollen for their broods that they do not serve their host plants as well as other, less specialized, visitors (N. Williams and J. D. Thomson, unpublished manuscript).

\section{Putting pollination deficits into the context of plant life histories}

In wild populations where conservation is an issue, we must ask whether pollination deficits are likely to affect demography and persistence. This is most important in long-lived perennials, because increased fecundity may incur costs of reproduction that counteract potential gains. Very few studies have explicitly tied pollination limitation to population trajectories (Bierzychudek 1982, Calvo 1993, Ehrlén and Eriksson 1995, Parker 1997), and these studies present a complicated picture. Ehrlén and Eriksson (1995) found that supplementally pollinated plants of Lathyrus verna increased their seed production by 3.1 times, but then shrank in size, made fewer flowers the next year, and were more likely to enter dormancy than were controls. The overall consequences for estimated population growth rates were negligible: $(\lambda=1.02$ for supplemented plants and $\lambda=1.03$ for controls).

In contrast, Parker (1997) found that strong pollination limitation of seed production in the invasive weed Cytisus scoparius did affect recruitment: costs of reproduction in this species were not strong enough to cancel out the value of extra seed production. Interestingly, populations of $C$. scoparius that experienced stronger pollination deficits had higher growth rates and were more invasive than those populations where pollinators were more abundant. This surprising effect arose because the habitats with more pollinators had extremely depressed seed germination. This example indicates that cross-habitat comparisons of pollination deficits may provide misleading diagnoses of where a plant species is in trouble. 


\section{Does life-history evolution set up patterns that serve as signatures of pollinator declines?}

The study by Ehrlén and Eriksson (1995) is consistent with the idea that perennial plants evolve toward an optimal allocation of resources to various functions, given a set of prevailing environmental conditions. One of those environmental conditions would be the expected level of pollination. In principle, ecologically abrupt changes in pollination level, such as a drop caused by pollinator declines, would disrupt this balance until natural selection had time to retune it. Such disruptions might, therefore, produce a syndrome of reproductive characteristics that would signal a diminished pollination regime. These would include chronically depressed fecundity, not just the occasional seasons of pollination limitation that we expect from Haig and Westoby's argument (1988), coupled with increased vegetative vigor, such as growth rates or clonal spread, or possibly increased flower production or longevity. In practice, these characteristics are likely to be so plastic and so influenced by other factors that reliable inferences may be hard to draw except in certain situations, such as comparing the performance of a species in different habitats. Even in that situation, one would also need to intervene experimentally to show that observed differences in, say, clonal expansion are due to pollination levels rather than to other characteristics of the habitats.

\section{ELEMENTS OF A THOROUGH STUDY OF POLLINATION DEFICITS}

I conclude with a case study from Japan that has linked an apparent shortfall in fecundity to a pollination deficit caused by a past unobserved decline in pollinator populations. Although the investigation of Primula sieboldii by Izumi Washitani and her colleagues is not absolutely airtight and complete, it is the best study I know (Washitani et al. 1991, 1994a, b, Washitani 1996; I. Washitani, personal communication).

Primula sieboldii is a clonal, distylous herb with deep-tubed flowers that seem adapted for pollination by long-tongued bees. The plant is rare enough to be a conservation target. A population at one small nature reserve (Tajimagahara, on the densely populated principal island of Honshu) appeared to have very poor seed set, which Washitani hypothesized was the result of inadequate pollination. To retain this hypothesis, it would, of course, be necessary to reject some alternative explanations, such as the possibility that other conditions, such as pollution or soil infertility, had rendered the site inimical to seed production. In this as in other Primula spp., rare autogamous, homostylous individuals occur in which anthers and stigmas are borne at the same level and the self-incompatibility reaction breaks down. At Tajimagahara, these self-pollinating, homostylous clones set seed freely at levels well beyond those of the typical pins and thrums that require outcrossing by animals. Therefore, adverse physical conditions could be ruled out. Furthermore, many hours of filming flowers in the reserve revealed no insect visits.

In the Hidaka region of Hokkaido, numerous $P$. sieboldii populations occur in forest fragments separated by a matrix of agricultural land (mostly pasture). Some, but not all, of these fragments support populations of the long-tongued bumble bee Bombus diversus tersatus. Queens are active at the time of $P$. sieboldii bloom: they visit the flowers and carry pin and thrum pollens spatially segregated on the proboscis in accordance with the classic picture of distyly. When a bumble bee visits a flower of this species, its tarsal claws puncture the petals and produce characteristic whitish lesions that are easily scored. Across forest fragments, seed set was significantly correlated with the frequency of flowers bearing claw marks. Furthermore, seed set was significantly increased by introducing a $B$. diversus queen into a flight cage erected in the field over pin and thrum clones. Cumulatively, these pieces of evidence make a strong case for true pollination deficits that are attributable to a dearth of pollinators; that dearth is almost certainly the result of a pollinator decline in some of the forest remnants that are less suitable for maintaining bumble bee populations. It is important to learn more about the characteristics that cause some patches to support bees while others do not; it is also important to reintroduce bees to patches where they are currently absent. Nevertheless, the index of bee activity provided by the claw marks makes it possible to draw strong inferences that could not be made without some estimate (or manipulation) of bee abundance.

\section{SUGGESTIONS FOR FUTURE RESEARCH}

Documenting population declines of pollinators, especially insects, is so hard to do that there are hardly any case studies. In comparison, measuring pollination deficits of plants is easy, and there are dozens of studies. I argue that making measurements of this type should be part of any effort in plant conservation where pollinator declines might be a concern. Nevertheless, deficit estimates themselves illuminate only one aspect of a complex problem. Pollinator shortages and seed shortfalls are functionally related, of course, but only under certain restrictive circumstances is it safe to infer a past decline from a present deficit. Evolutionary theory suggests that plants should allocate resources so that pollination deficits are occasional rather than chronic; therefore, chronic deficits might plausibly indicate recent declines in pollinator service. However, this must be regarded as a weak prediction. Such inferences can be strengthened by comparing the levels of deficits across different habitats that might differ in terms of pollinator density, such as insecticide-sprayed sites vs. control sites, isolated fragments vs. continuous habitat, etc. A better design would enrich such habitat comparisons with actual measurements of pollinator abundance, as in Washitani's work, and the best design would include direct manipulations of pollinator populations. 


\section{RESPONSES TO THIS ARTICLE}

Responses to this article are invited. If accepted for publication, your response will be hyperlinked to the article. To submit a comment, follow this link. To read comments already accepted, follow this link.

\section{Acknowledgments:}

This paper resulted from an NSF-funded workshop conducted at the National Center for Ecological Analysis and Synthesis, a Center funded by NSF (Grant \# DEB 94-21535), the University of California at Santa Barbara, and the State of California. I particularly thank Jim Cane, Karen Goodell, Vince Tepedino, Barbara Thomson, Izumi Washitani, Neal Williams, and Paul Wilson.

\section{LITERATURE CITED}

Bell, G. 1985. On the function of flowers. Proceedings of the Royal Society of London B, Biological Sciences 224 : 223-265.

Bierzychudek, P. 1982. The demography of jack-in-the-pulpit, a forest perennial that changes sex. Ecological Monographs 52: 335-351.

Buchmann, S. L., and G. P. Nabhan. 1996. The forgotten pollinators. Island Press, Washington, D.C., USA.

Burd, M. 1994. Bateman's principle and plant reproduction: the role of pollen limitation in fruit and seed set. Botanical Review 60: 83-139.

Calvo, R. N. 1993. Evolutionary demography of orchids: intensity and frequency of pollination and the cost of fruiting. Ecology 74: 1033-1042.

Charnov, E. L. 1982. The theory of sex allocation. Princeton University Press, Princeton, New Jersey, USA.

Ehrlén, J., and O. Eriksson. 1995. Pollen limitation and population growth in a herbaceous perennial legume. Ecology 76: 652-656.

Goodell, K., and J. D. Thomson. 1997. Comparisons of pollen removal and deposition by honey bees and bumble bees visiting apple. Acta Horticulturae 437: 103-107.

Haig, D., and M. Westoby. 1988. On limits to seed production. American Naturalist 131: 757-759.

Harder, L., and J. D. Thomson. 1989. Evolutionary options for maximizing pollen dispersal of animal-pollinated plants. American Naturalist 133: 325-334.

Kearns, C. A., D. W. Inouye, and N. M. Waser. 1998. Endangered mutualisms: the conservation of plantpollinator interactions. Annual Review of Ecology and Systematics 29: 83-112.

Mayfield, M. M. 1998. Shortcomings of current crop pollination strategies: lessons from natural pollination systems. Thesis. Reed College, Eugene, Oregon, USA.

McGregor, S. E. 1976. Insect pollination of cultivated crop plants. Agricultural Handbook Number 496, Agricultural Research Service, U.S. Department of Agriculture, Washington, D.C., USA.

Parker, I. M. 1997. Pollinator limitation of Cytisus scoparius (Scotch broom), an invasive exotic shrub. Ecology 78 : 1457-1470.

Tepedino, V. J., S. D. Sipes, and T. L. Griswold. 1999. The reproductive biology and effective pollinators of the endangered beardtongue Penstemon penlandii (Scrophulariaceae). Plant Systematics and Evolution 219: 39-54.

Thomson, J. D., and B. A. Thomson. 1992. Pollen presentation and viability schedules in animal-pollinated plants: consequences for reproductive success. Pages 1-24 in R. Wyatt, editor. Ecology and evolution of plant reproduction: new approaches. Chapman and Hall, New York, New York, USA.

Washitani, I. 1996. Predicted genetic consequences of strong fertility selection due to pollinator loss in an isolated population of Primula sieboldii. Conservation Biology 10: 59-64.

Washitani, I., M. Kato, J. Nishihiro, and K. Suzuki. 1994a. Importance of queen bumble bees as pollinators 
facilitating inter-morph crossing in Primula sieboldii. Plant Species Biology 9: 169-176.

Washitani, I., H. Namai, R. Osawa, and M. Niwa. 1991. Species biology of Primula sieboldii for the conservation of its lowland-habitat population. (1). Inter-clonal variations in the flowering phenology, pollen load and female fertility components. Plant Species Biology 6: 27-37.

Washitani, I., R. Osawa, H. Namai, and M. Niwa. 1994b. Patterns of female fertility in heterostylous Primula sieboldii under severe pollinator limitation. Journal of Ecology 82: 571-579.

Willson, M. F. 1979. Sexual selection in plants. American Naturalist 113: 777-790.

Wilson, P., and J. D. Thomson. 1991. Heterogeneity among floral visitors leads to discordance between removal and deposition of pollen. Ecology 72: 1503-1507.

Wilson, P., J. D. Thomson, M. L. Stanton, and L. P. Rigney. 1994. Beyond floral Batemania: gender biases in selection for pollination success. American Naturalist 143: 283-296.

Young, H. J. 1988. Differential importance of beetle species pollinating Dieffenbachia longispatha (Araceae). Ecology 69: 832-844.

Young, H. J., and T. P. Young. 1992. Alternative outcomes of natural and experimental high pollen loads. Ecology 73: 639-647.

\section{Address of Correspondent:}

James D. Thomson

Department of Zoology

University of Toronto

Toronto, Ontario, Canada M5S 3G5

Phone: 416-978-3500

Fax: $416-978-3542$

jthomson@zoo.toronto.edu 